\title{
La correspondance des Marcille adressée aux Goncourt
}

Dominique Pety

\section{Citer ce document / Cite this document :}

Pety Dominique. La correspondance des Marcille adressée aux Goncourt. In: Cahiers Edmond et Jules de Goncourt n9, 2002. pp. 239-278;

doi : https://doi.org/10.3406/cejdg.2002.901

https://www.persee.fr/doc/cejdg_1243-8170_2002_num_1_9_901

Fichier pdf généré le 17/05/2018 


\section{La correspondance des Marcille adressée aux Goncourt}

Les 30 épais volumes in $-4^{\circ}$ de la Correspondance adressée aux Goncourt conservés à la Bibliothèque nationale de France ${ }^{1}$ sont une ressource essentielle pour qui veut mesurer le rayonnement intellectuel et culturel des Goncourt en leur temps. Ils ont été partiellement exploités pour l'établissement de correspondances croisées (avec ref "Huysmans (J.-K.) " Henri Céard, Daudet ref " Daudet (A. et J.) ", Flaubert ref "Flaubert (G.) »). Mais les masses d'ombres qui demeurent sont énormes ${ }^{2}$.

En 1968, Jean Adhémar avait révélé aux historiens de l'art le profond intérêt des lettres d'artistes, d'amateurs, d'administrateurs de musées dans ces volumes ${ }^{3}$. Quelques extraits significatifs en furent ainsi publiés à l'occasion de monographies (voir le catalogue Chardin de 1979 qui cite des lettres de collectionneurs, l'ouvrage de Weisberg sur P. Burty ref "Burty (P.) ", etc.). Parmi les nombreux collectionneurs, bibliophiles, éditeurs, critiques d'art, hommes des musées et des bibliothèques avec lesquels les Goncourt étaient en relation ${ }^{4}$, nous avons choisi de présenter ici deux figures de premier plan : Eudoxe et Camille Marcille. Les lettres adressées aux Goncourt par les deux frères Marcille, mais aussi par $\mathrm{M}^{\mathrm{me}}$ Camille Marcille et ses

1. Cabinet des manuscrits NAF 22450-22479) (30 vol.).

2. Pierre Dufief, qui a publié en volumes les lettres de Flaubert et Daudet (assorties des réponses des Goncourt qui, elles, ne figurent pas dans ces volumes de la $\mathrm{BnF}$ ) et, dans les Cahiers Goncourt, les lettres de Barrès, Montesquiou, Nadar et du cousin E. Labille, travaille au projer d'une édition intégrale.

3. "I ertres adressées aux Goncourt, concernant lcs beaux-arts, cunservées à la Bibliothèque nationale ", Gazette des Beaux-Arts, $110^{\mathrm{e}}$ année, 6e période, vol. 72, 1968, p. 229-236.

4. Pour le seul volume XVIII (NAF 22467 Labarre-Letellier), on peut citer les noms de collectionneurs comme le comte de La Béraudière, le marquis de Laborde, Louis La Caze, Laurent 
filles ${ }^{5}$, témoignent d'une part de relations suivies avec des collectionneurs renommés de l'art du XVIII siècle, d'autre part, des liens d'affection établis par les deux frères avec toute une famille. On découvre ainsi un autre relief à la vie culturelle du XIX $\mathrm{XIècle} \mathrm{et} \mathrm{à}^{\mathrm{c}}$ la vie personnelle des Goncourt, que le Journal, malgré sa richesse, ne permet que d'entrevoir. Les lettres des frères Marcille donnent des renseignements précis sur leurs propres collections, mais aussi sur le rôle que pouvait jouer, au XIX ${ }^{\mathrm{e}}$ siècle, un collectionneur dans l'administration des musées de province ou l'organisation d'expositions ${ }^{6}$. Celles de $\mathrm{M}^{\mathrm{me}}$ Marcille et de ses filles, si belles de sincérité et d'affection, sont d'un grand prix sur le plan humain. Elles contredisent l'étiquette de misogynes et de misanthropes qu'on a si souvent attribuée aux Goncourt, en s'appuyant sur les maximes expéditives du Journal; elles gratifient le chercheur dans ses enquêtes parfois fastidieuses en lui restituant la fraîcheur des mots d'enfants : les Goncourt, en conservant soigneusement ces lettres reçues ${ }^{7}$, avaient probablement conscience de constituer pour le siècle futur une réserve de documents autographes, dont ils avaient eux-mêmes goûté le bonheur de la vie restituée.

Eudoxe (1814-1890) et son cadet Camille (1816-1875) sont les fils du grand collectionneur François Marcille (1790-1856) ${ }^{8}$. Ce dernier est l'un des quatre enfants d'un bourgeois d'Orléans, issu d'un milieu modeste de cultivateurs, mais enrichi dans le commerce des

Laperlier, Leber, His de La Salle ísans comprer des particuliers moins illustres qui proposent ponctuellement un renscignement, un document, aux deux frères dont la notoriété de spécialistes s'affirme) ; parmi les bibliophiles, Paul Lacroix, parmi les éditeurs, Albert Lacroix, dans l'administration des Beaux-Arts, Gustave Larroumet...

5. NAF 22 469, vol. XX, Marcille-Masson.

6. Comme l'a montré M.-C. Genêt-Delacroix, ce n'est qu'à la fin du siècle qu'apparaît un personnel spécialement formé à ce type de gestion ("État et patrimoine sous la III République : de l'amateur au professionnel dans la gestion du patrimoine national ", p. 147-160 dans L'Esprit des lieux. Le patrimoine et la cité, sous la direction de Daniel J. Grange et Dominique Poulot, Presses universitaires de Grenoble, 1997).

7. Journal, 22 janvier 1890 : "Je rangeais mes lettres de l'année dernière et parmi les lettres admiratives d'enthousiastes de ma littérature [...]".

8. Sur F. Marcille, voir Philippe Huisman, "La collection Marcille : 5000 tableaux méconnus ", Connaissance des arts, $\mathrm{n}^{\circ} 88$, juin 1959, p. 74-81. Cet article ajoute des précisions aux portraits 
laines, et qui participe en notable, entre 1815 et 1830 , aux assemblées politiques de la ville. François, qui devait succéder à son père à la tête du négoce, préfere dessiner, peindre, courir les rares brocanteurs d'Orléans, parcourir le Musée. En 1822, après son mariage', et alors que ses propres fils ont huit et six ans, il peut, grâce à une petite rente mensuelle de son père, s'installer à Paris, où ses goûts d'artiste et d'amateur l'ont attiré ${ }^{10}$. Il étudie les toiles de maîtres au Louvre; et, comme le musée ferme tôt, il emprunte le soir à un marchand des têtes de Greuze pour les copier ${ }^{11}$; il déniche chez un autre tout un carton de dessins de Prud'hon ${ }^{12}$. À la vente Poterlet (1840), il acquiert des toiles du même, et de Watteau, Chardin, Boucher, Latour et Greuze. Il effectue aussi des voyages en Italie, en Belgique et en Hollande, et acquiert des toiles d'écoles étrangères. Le XVIII ${ }^{\mathrm{e}}$ s., pour ses bas prix et parce qu'il est encore à redécouvrir, reste sa préférence. Il a pour amis His de La Salle, Walferdin, et surtout La Caze, avec qui il fréquente quotidiennement l'hôtel des ventes, alors rue des Jeûneurs.

Eudoxe et Camille, après les cours du collège Stanislas, reçoivent une formation de peintres, dans l'atelier de Steuben, puis auprès d'Achille Devéria, et font, en 1839, un premier voyage d'études en Allemagne et en Italie ${ }^{13}$. À l'occasion de son mariage avec une Orléanaise, Mlle Erat-Oudet, qui mourra très jeune, son père donne à Eudoxe un petit lot de tableaux, qu'il complétera ensuite avec ses

\footnotetext{
brossés par G. Duplessis et $\mathrm{H}$. de Chennevières (art. cités ci-après), mais sans en indiquer les sources. Il donne en outre un bon aperçu de l'évolution des prix de l'arr du XVIII's. au temps des Marcille : "Un Chardin payé quelques francs vers 1830 [par F. Marcille] atteignait déjà quelques centaines de francs en 1856 [vente de F. Marcille] et quelques milliers de francs vingt ans plus tard à la vente après décès de C. Marcille. En 1912, à la vente Jacques Doucet, on vendait une ceuvre du maître 300000 francs or. "

9. Il s'est marié en 1813 avec la petite-fille du maire d'Étampes, Simonneau, tué par les émeutiers en 1793.

10. Il habite d'abord rue Bourbon, puis il déménagera pour un logement plus grand, rue de Tournon. 11. Alphonse Giraud, papetier, a adjoint à sa boutique une galerie de peinture, constituée surtout des tableaux et dessins rachetés à la mort de Greuze dans son atelier.

12. C'cst lc bouquiniste et marchand d'estampes Dauvin, rue Bonaparte.

13. Paul Ratouis de Limay, "Trois collectionneurs du XIX`s. His de La Salle. Le docteur La Caze. Les Marcille. III. La collection des Marcille ", Le Dessin, Revue d'art, d'éducation et d'enseignement, $10^{\circ}$ annéc, $n^{\circ} 6$, décembre 1938 , p. 304-315.
} 
propres acquisitions. Dès 1846 , il habite dans l'hôtel de ses beauxparents, rue d'Hauteville, un atelier-galerie. Deux voyages en Italie et un séjour à Londres en 1854 en compagnie de La Caze complètent sa formation d'artiste et d'amateur. Camille aura quant à lui son atelier rue Madame, puis rue Monsieur-le-Prince, où il est voisin du paysagiste Aligny. En février 1854, il épouse Cécile Walckenaer, petitefille du baron Walckenaer ${ }^{14}$, et s'installe à Oisème, une propriété qu'il a acquise près de Chartres. En septembre 1856, François Marcille, malade, appelle ses fils à son chevet et partage entre eux les plus belles pièces de sa collection. Il meurt en novembre. Le reste est dispersé en trois ventes successives (tableaux anciens des écoles françaises, italiennes, hollandaises et flamandes : $12-17$ janvier, $628 \mathrm{n}^{\text {os }} ; 2-3$ mars, $300 \mathrm{n}^{\text {os }}$; dessins, esquisses et estampes (plus de 4000) : 4-7 mars 1857).

Camille emporte à Oisème sa part (13 tableaux de Chardin, 9 toiles et 55 dessins de Prud'hon, 2 peintures de Géricault, 2 tableaux de Fragonard, 1 peinture et 3 études peintes de Marilhat, plusieurs Greuze, Lancret, Boucher, Hubert Robert, Desportes ${ }^{15}$...). Sa collection, comme celle de son frère, concerne essenticllement l'école française du XVIII ${ }^{e}$ et du début du XIX ${ }^{e}$ siècle, mais est également ouverte à des artistes d'autres temps et d'autres pays. Dans sa retraite

14. Charles Athanase, baron Walckenaer (1771-1852). Après des études à Paris, poursuivies à Oxford et Glasgow, il revient en France (1791), où il est pendant 18 mois directeur des transports de l'armée des Pyrénées; soupçonné de modérantisme, il préfere abandonner sa place. Il reçoit en 1811 le prix de l'Institut pour la question "Quels ont été les peuples qui ont habité les Gaules Cisalpine et Transalpine avant l'année 410 de J. C., l'étendue de leur territoire et l'emplacement de leur capitale?". En 1813, il devient membre de l'Institut. En 1814, il reçoit de Louis XVIII la croix de la Légion d'honneur. Il devient en 1816 secrétaire général de la préfecture de la Seine, puis maire du Vearrondissement. Il est nommé préfet de la Nièvre en 1826, et de l'Aisne en 1828. Révoqué en 1830, il obtient en 1839 l'emploi de trésorier de la bibliothèque royale et de conservateur des cartes géographiques. Légitimiste, il reste éloigné des luttes de partis et se consacre surtout à l'étude : entomologie (Histoire naturelle des arachnéides, 1805-1808), géographie (Tableau géographique et historique de la Polynésie et de l'Australie, 1819, 4 vol.; Recherches géographiques sur l'Afrique septentrionale, 1821; Géographie ancienne, historique et comparée des Gaules cisalpine et transalpine, 1839, 3 vol.), biographie littéraire (Histoire de la vie et des ouvrages de La Fontaine, 1820 ; Histoire de la vie et des poésie d'Horace, 1840, 2 vol., Mémoires sur M'me de Sévigné, 18441852,5 vol., inachevé).

15. Georges Duplessis, "La collection de M. Camille Marcille ", Gazette des Beaux-Arts, 1 cr mars 1876 , p. 419-439. 
provinciale, Camille agrandira peu l'héritage paternel; il s'occupe surtout de peindre, et expose à plusieurs Salons (entre 1859 et 1870) des fleurs et des scènes de genre. Il est nommé en 1862 directeur du Musée de Chartres, pour lequel il fera plusieurs acquisitions. Avec l'aide de sa femme, qui a elle-même des goûts d'art, il accueille chez lui " tout le Louvre de l'époque : Soulié, Clément de Ris, P. de Chennevières, et les Goncourt, et les critiques, et les gens de lettres d'un peu d'érudition ${ }^{16}$ ". Il meurt le 3 août 1875 , et en dépit des prix élevés de sa vente, son frère rachète le plus d'œuvres possible.

Eudoxe, à l'inverse de Camille, sera plus collectionneur que peintre, plus sollicité à Paris pour acquérir ${ }^{17}$. En 1862, vient la reconnaissance officielle de l'amateur : Nieuwerkerke le nomme membre du jury des Salons, ce qu'il sera jusqu'en 1878 (section de la gravure, puis de la peinture, en compagnie notamment de La Caze, du marquis Maison, de Théophile Gautier, de Paul de Saint-Victor, de Frédéric Reiset). En avril 1870, il est nommé conservateur du Musée d'Orléans; il en établit le catalogue, jusqu'alors inexistant (il parâ̂tra en 1876), et se montre incessamment attaché à son accroissement, achetant sur ses propres fonds, ou sollicitant en vue de donations collectionneurs de la région ou artistes natifs d'Orléans. Président de la Société des Amis des arts d'Orléans, qui organise des séances de musique et de lecture, directeur de l'École gratuite de dessin, il anime la vie culturelle locale, tout en restant très actif à Paris. Il organise notamment en 1874 une exposition de l'œuvre de Prud'hon pour venir en aide à sa fille dans la misère, et sollicite à ce propos les collectionneurs (mais son frère et lui-même sont les plus gros prêteurs). Il organise d'autres expositions (Diaz, Fromentin), et entretient d'excellentes relations avec la Conservation des peintures et des dessins du Louvre, signalant des occasions, servant même quelquefois d'intermédiaire dans les ventes publiques; il deviendra Membre du conseil des musées. Un conservateur du Louvre écrira à son propos: "Il faut faire litière du vieux mot fané de mécène.

16. Henry de Chennevières, "Silhouettes de collectionneurs. M. Eudoxe Marcille ", Gazette des Beaux-Arts, $1^{\text {cr }}$ septembre 1890 , p. 217-235, et $1^{\text {cr }}$ octobre 1890 , p. 296-310.

17. L'Annuaire des artistes et des amateurs 1862 de Paul Lacroix (Renouard, 1862) fait l'éloge d'Eudoxe Marcille, qui aurait reconstitué la splendeur de la collection paternelle (Horsin Déon, « Notice sur les cabinets d'amateurs à Paris. M. Eudoxe Marcille ", p. 128-138). 
Quand on parle devant nous d'un véritable ami des arts, disons : $c^{\prime}$ est un Marcille ${ }^{18}$.

Les Goncourt rencontrent Eudoxe Marcille en 1858. Du moins le Journal relate-t-il leur première visite au collectionneur à la date du 29 avril 1858 :

Que de Prud'hon chez un des deux fils de M. Marcille! Il en tire des murs, des armoires, des coffres, de partout. C'est le chapeau de Fortunatus : dessins, académies, tableaux... Un grand homme, Prud'hon, et un très bon homme, l'autre!

Eudoxe les emmène ensuite à Chartres, le 28 mai ("Nous partons avec Eudoxe Marcille pour voir une exposition de tableaux et de dessins à Chartres. Tout l'intérêt de cette exposition est dans les Prud'hon et les Chardin appartenant à $M$. Camille Marcille, frère de M. Eudoxe "). Ils font alors probablement connaissance de Camille Marcille et de sa famille. Le 8 mai 1859, Eudoxe les présente au Docteur la Caze, dont ils découvrent la collection : on voit comment se tissent ainsi, à la fin des années 1850, les relations entre les Goncourt et des collectionneurs de renom; ils commencent à être reconnus par leurs pairs, ce qui leur permettra probablement de figurer comme prêteurs à l'Exposition de 1860 du boulevard des Italiens. Mais ils n'ont pas encore le zèle d'un Eudoxe, dont ils ne peuvent en 1860 comprendre l'intérêt presque exclusif pour un peintre :

Je vois Marcille : un homme dont toute la vie est de savoir s'il aura pour 2000 francs une miniature de Prud'hon, Mademoiselle Mayer, avec une levrette en camaïeu au-dessus. Vraiment, se consacrer à une mémoire de cette façon-là, c'est être quelque chose comme le laquais d'une immortalité '14 janvier 1860].

Il est vrai qu'ils ne cesseront de se vouloir à la fois amateurs d'art et hommes de lettres; mais la monomanie de tout collectionneur, d'abord dénoncée par les deux frères, Edmond la reprendra volontiers à son compte après 1870 , pour attester de l'authenticité de ses goûts, alors que les modes s'en mêlent; à un moment aussi où, solitaire et livré à ses propres penchants, il commence à acquérir en des

18. Philippe Huisman, art. cit., p. 81. 
proportions nouvelles (mais il s'agit désormais d'objets japonais).

La première invitation à Oisème date de l'automne 1863 (c'est l'objet de la première lettre de Camille et d'Eudoxe que nous présentons ici). Y répond la description par les Goncourt de leur séjour, dans le Journal (16-19 octobre 1863). Ils s'y montrent enchantés du chaleureux accueil, particulièrement de l'affection des fillettes, et sensibles aussi au charme de $\mathrm{M}^{\text {me }}$ Marcille. Ils les revoient à Paris, en décembre, chez Eudoxe, où elles sont venues passer quelques jours (voir Camille, lettre 3; Eudoxe, lettre 1, et Journal, 17 décembre 1863, où Edmond commence à évoquer, mais au conditionnel, l'amour qu'il porte à $\mathrm{M}^{\text {me }}$ Marcille). Suit en janvier 1864 l'envoi d'une caisse de jouets pour les étrennes des enfants (voir $\mathrm{M}^{\mathrm{mc}}$ Marcille, lettre 1, et Mlles Marcille, lettre 1). La correspondance et les rencontres doivent se poursuivre ensuite assidûment : $\mathrm{M}^{\mathrm{me}}$ Marcille appelle désormais par jeu Jules de Goncourt " mon gendre" (et non plus " mon cher Monsieur"), parce qu'il semble être le compagnon privilégié de sa fille Juliette; et Edmond poursuit ses confidences voilées dans le

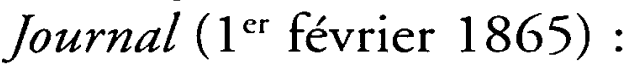

En recevant une lettre de la petite Marcille, une de ces jolies lettres comme les enfants en trouvent sous la dictée de leur mère, je pensais à une jolie introduction de roman d'amour. Ce serait l'amour d'une femme du monde, caché sous les doigts de son bébé.

Pendant ce temps, à Paris, les relations avec Eudoxe se poursuivent : les Goncourt lui procurent, en 1864, une première invitation chez la princesse Mathilde, où ils sont quant à eux reçus depuis 1862 (Eudoxe, lettres 2 et 3).

Le Journal décrit, à la date du 27 juin 1865, un autre séjour à Oisème, tout occupé de jeux avec les enfants, et qui donnera lieu à deux lettres lourdes de regrets après le départ des deux frères (voir $\mathrm{M}^{\text {me }}$ Marcille, lettre 3, Mlles Marcille, lettre 2). Mais, en avril 1868, de nouveau invités par Eudoxe à venir dîner chez lui pour y rencontrer Camille et sa famille (voir Eudoxe, lettre 4), ils reçoivent une mise une garde de $\mathrm{M}^{\mathrm{me}}$ Marcille, dont le mari témoigne de la jalousie (Journal, 23 avril 1868). Et les relations cessent probablement.

La mort de Jules les renoue : $\mathrm{M}^{\text {me }}$ Marcille envoie ses condoléances dans une lettre pleine de tendresse (lettres 5 et 6,23 juin et 2 juillet 1870). En outre, Edmond, pour ses travaux d'érudition qui lui per- 
mettront de renouer avec l'écriture après la mort de son frère, sollicite d'Eudoxe et de Camille des renseignements sur les œuvres qu'ils possèdent. Inversement, Eudoxe demande à Edmond une information pour la rédaction de son catalogue du Musée d'Orléans, ou le prêt d'un dessin pour l'exposition consacrée à Prud'hon. À la mort de Camille, que lui annonce Eudoxe (lettre 9, du 4 août 1875), Edmond prête son concours pour l'organisation de la vente, qui sera un succès (voir $\mathrm{M}^{\text {me }}$ Marcille, lettres 13 et 14 ). Les relations avec $M^{\text {me }}$ Marcille se poursuivront jusqu'au milieu des années 1885 , teintées de la mélancolie qui les imprègne depuis la mort de Jules; viendront s'y ajouter la mort de Camille, et la longue maladie de la petite Jane.

Dominique PETY

\section{CAMILLE MARCILLE}

1. [f. 3]"

Messieurs,

Mon frère est arrivé à Oisème où il doit rester jusqu'au 10 novembre. Ça serait bien le moment de venir prendre des notes sur Chardin que nous mettons tous au-dessus de Raphaël puisqu'il doit vous attirer dans notre vallée.

Veuillez songer, Messieurs, que j'ai fait construire au-dessus de l'atelier des chambres d'où l'on découvre la prairie encore très verte, et surtout pensez au plaisir que vous nous ferez à tous en restant longtemps parmi nous.

Quand votre voyage sera fixé, soyez assez bons messieurs pour me prévenir afin que j'aille vous chercher au débarcadère, et veuillez agréer l'assurance de notre sincère dévouement.

Oisème 7 octobre [1863]

C. Marcille

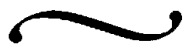

19. Pour chaque correspondant, les feuillets sont numćrotés f. 1, f. 2, etc. Le feuillet classé en troisième position parmi les envois de Camille Marcille est en fait le premier adressé aux Goncourt. 
2. $[\text { f. } 4]^{20}$

\section{Chers Messieurs,}

Je suis heureux de pouvoir vous dire que le plaisir que mon frère éprouvera à vous voir sera partagé par moi. Venez, je vous prie, à Oisème : vous y serez bien reçus : et vous serez contents de l'atelier qui renferme quelques œuvres de vos artistes favoris. Venez prendre les notes dont vous avez besoin pour faire votre article sur Chardin. En partant à 7 heures 30 , à 8 heures ou à 10 heures 40 de la gare Mont Parnasse vous serez deux heures après à Chartres, où je vous attendrai, chers Messieurs, tout disposé à vous être agréable.

Veuillez agréer l'assurance de mon affectueux dévouement.

78 bre 1863

Eud. Marcille

Oisème près Chartres

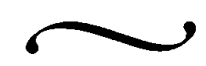

3. [f. $1-2]^{21}$

\section{Chers Messieurs,}

Grands et petits ont vivement regretté votre départ et tous aspirent à vous revoir à Oisème. Je vous aurais écrit cela il y a deux jours après avoir reçu votre aimable lettre, mais j'ai appris en même temps que votre article sur Chardin avait paru dans la Gazette des Beaux$A_{r t 5^{22}}$, et j'ai voulu le lire et le relire à ces dames. Il me serait difficile de vous répéter tous les éloges qu'on en a fait. Votre modestie ne voudrait voir dans les miens que la satisfaction de l'amateur; mais comme il y a trente ans que j'aime Chardin, je puis dire librement que votre portrait est parfait et que vous avez accroché l'auréole à la tête de votre saint.

20. Lettre écrite sur la même feuille que la précédente, sur la deuxième page que forme cette feuille pliée en deux.

21. Cette lettre, placée en première position, est postérieure aux deux précédentes : la monographic Chardin, en préparation, est ici achevée.

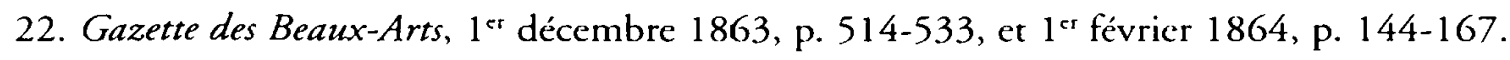


Ma femme part aujourd'hui pour Paris avec ses trois démons; je reste avec ma belle-sœur et ses enfants qui ne peuvent oublier votre inaltérable complaisance.

Veuillez, chers Messieurs, ne conserver le souvenir d'Oisème que pour y revenir et faire des heureux.

Votre tout dévoué

Oisème 10 décembre [1863]

C. Marcille

4. [f. 5-6]

$[1873 \text { ou } 1874]^{23}$

Cher Monsieur,

Vous ne deviez pas être averti par tout autre que nous du mariage de Juliette, et en effet dès que cette grande détermination a été prise, ma femme vous a écrit. Cette lettre sans réponse ne nous faisait pas douter, cher Monsieur, de l'intérêt que vous prendriez à cet événement et votre bienveillant souvenir nous prouve ce matin que la nouvelle ne vous était pas parvenue.

Dans cette question nous n'avons fait qu'approuver le choix de notre fille. Les renseignements si favorables sur la famille du futur et la sympathie générale dont il jouit lui-même nous ont fait prendre une prompte décision.

Agréez, je vous prie, cher Monsieur, nos plus affectueux remerciements pour les voux que vous nous adressez. La jeune fiancée me charge de vous dire qu'elle ne peut oublier les jours que vous avez passés au milieu de nous et qui j'espère reviendront.

C. Marcille

Voici la liste de mes tableaux de Chardin (natures mortes).

Deux ovales faisant pendant. L'un représente un melon coupé sur-

23. Edmond complète les monographies de L'Art du XVIII' siècle par un dernicr fascicule de nores qui paraîtra séparément en 1875, mais qui est déjà intégré à l'édition Rapilly de 1873-1874, ce qui peut nous permettre de dater approximativement cette lettre. 
monté de la tranche, un panier de pêches, 3 prunes de reine Claude, 2 poires, un pot à eau de Chine et la cuvette plus deux bouteilles. Répétition de celui du musée La Cazexe "La Caze (L.) ".

Dans l'autre un bocal 2 abricots deux biscuits un citron un macaron et un couteau à manche noir qui rompt la ligne d'une tablette en marbre qui supporte en outre une boîte de dragées un pain de sucre recouvert de son papier bleu 2 tasses du Japon (blanc laiteux avec fleurs rouges) et 3 verres dont l'un est rempli de vin.

Ces deux tableaux sont signés avec la date de 1760. Ils furent achetés par mon père chez un descendant de Chardin qui demeurait derrière la porte $\mathrm{St}[-]$ Denis ou St[-]Martin. J'opinerais plutôt pour la dernière. Haut. 0.57. Larg. 0.51.

Deux autres tableaux :

Un quartier de viande et deux oignons jetés sur une nappe, plus, une cruche en terre verte, un chaudron en cuivre et un grugeoir. Signé Chardin 1732.

Le pendant - un poulet un fromage de Hollande et 3 œufs. Les ustensiles de cuisine qui complètent le tableau sont la cruche verte le chaudron de cuivre et le grugeoir. Une raie et une botte d'oignons sont suspendus à la muraille. Le tableau est signé $\mathrm{S}$. Chardin sans date. $\mathrm{H}^{\text {eur }}$. 042. L. 033. Provenance inconnue.

Un vase de fleurs. Haut ${ }^{\text {cur }}$. 045 L. 37. Des œillets blancs et un rouge, des tubéreuses et des pois de senteur dans un vase de japon blanc avec des arabesques bleues. Sans signature. Il a été acheté par mon père dans les dernières années de sa vie, je ne sais à quel amateur.

[grand croquis au crayon du tableau] ${ }^{24}$

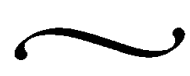

5. [f. 8-9]

Cher Monsieur,

Je vous envoie les renseignements que vous me demandez tout en regrettant que vous ne puissiez venir les prendre vous-même, mais nous espérons bien que ce qui est différé n'est pas perdu. 
142. Portrait de la $B^{\text {onne }}$ de Talleyrand. $\mathrm{H}^{\mathrm{r}}$. $0.24 \mathrm{~L}^{\mathrm{r}}$. 0.16 dessin sur papier bleu rehaussé de blanc, donné par [M.] de Talleyrand à son secrétaire que j'ai connu, mais dont je ne puis me rappeler le nom. La vente a été faite après sa mort, rue $\mathrm{St}[-]$ Dominique et le dessin a été payé 755 francs. Prud'hon a signé à droite. J'en fais la remarque parce que cette signature inachevée Prudh - est parfaitement originale. Si vous parvenez à savoir le nom du secrétaire, je saurai bien vous dire s'il est exact.

194. L'enlèvement de Psyché $\mathrm{H}^{\mathrm{r}}$. 0.60 L. 48 dessin sur papier bleu rehaussé de blanc très terminé pour servir au graveur. Acheté le 17 Décembre 1850, $2100[\mathrm{~F}]$ à la vente de [M.] Odiot père, en son hôtel sur l'emplacement du jardin Beaujon. C'est à cette même vente que mon père fit l'acquisition pour $995[\mathrm{~F}]$ de la petite esquisse pour le plafond du Louvre de la salle des antiques dite alors salle Laocoon (L'Étude donne l'essor au génie). Derrière l'esquisse est écrit : donné par l'artiste à [M.] Harassés ${ }^{25}$ (mal écrit - et peut-être mal interprété) $\mathrm{S}^{\text {re }}$ [secrétaire] général du musée Napoléon. À vérifier le nom dans les archives du Louvre ${ }^{26}$.

222. L'amour. Pastel sur toile H. 58 L. 48 étude pour le tableau dont j'ai l'esquisse L'innocence préfere l'amour à la richesse. Ce pastel acheté 300 francs avait été déposé chez Susse par le Marquis Maison qui par le même intermédiaire vendit $500[\mathrm{H}]$ à mon père le dessin intitulé La Philosophie, une des 4 figures qui avaient servi à la décoration de l'hôtel Lanois rue Laffitte. Le duc d'Aumale en a exposé ${ }^{*}$ pour les Alsaciens Lorrains ${ }^{27}$ deux autres. Qu'est devenu le $\left[4^{\mathrm{e}}\right]$ ? ${ }^{* 28}$

223. Le plaisir et l'innocence H. 37 L. 25 dessin sur papier blanc crayon noir et blanc. Étude d'après Julien et Marguerite pour le

25. Également illisible dans la lettre de C. Marcille.

26. Les deux descriptions des $n^{\circ} 142$ et 194 sont barrées de trois légers traits obliques à l'encre noire, peut-être des marques du travail d'Edmond de Goncourt, biffant des informations exploitées.

27. Exposition d'objets d'art au profit de la Société de protection des Alsaciens et Lorrains demeurés Français, dont l'initiative est née dans les salons de la haute socićté (voir A. Jacquemart, "Exposition en faveur de l'œuvre des Alsaciens et Lorrains demeurés Français ", Gazette des Beaux-

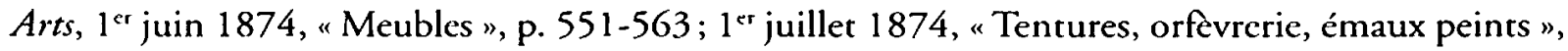

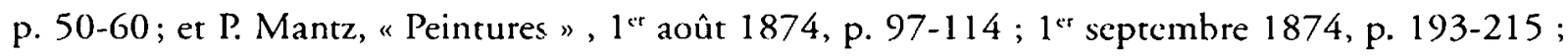
1'r octobre 1874 , p. 289-309).

28. Le passage entre les signes * est entouré légèrement à l'encre noire. 
tableau dont j'ai un petit croquis à la plume dans l'album que Prud'hon avait en Italie. Le dessin a été acheté 416 [F] le 8 décembre 1840 à la vente de Poterlet le père du peintre. Il y avait à cette vente plusieurs dessins de Prud'hon; ils sont dans la collection de mon frère. L'étude donne l'essor au génie dessin très terminé; un profil de femme, pour la mère heureuse ${ }^{29}$; plus un dessin à la plume que je possède.

224. Le repentir H. 32 L. 16 dessin sur papier blanc au crayon noir et blanc étude pour Le plaisir et l'innocence - vendu $1270[\mathrm{~F}]^{30}$ le 8 mai 1855 à la vente Renouard qui n'a rien de commun avec l'éditeur que le nom.

230. Themis. Je suppose que vous voulez parler de ce sujet où la victime est étendue au pied du tribunal. Première pensée du dessin du Louvre qui appartenait à Ledru Rollin. Le mien a été acheté 1 100 à la vente Revil. Hr. 26 L. 34. À propos du dessin du Louvre qui appartenait à Ledru Rollin en Angleterre le membre du gouvernement provisoire [,] alors aux expédients, envoya son ami David d'Angers proposer le dessin à mon père et lui en demanda $4000[\mathrm{~F}]$. Mon père lui en offrit 3000 . David alla de suite chez Jeanson alors directeur des beaux-arts qui conclut le marché.

231. Nemesis fragment sur papier bleu H. 26 L. 19 représente la déesse entraînant au tribunal le coupable et sa complice. Le peintre y substitua le témoin. Cette variante a été achetée plus tard à la première vente Carrier.

261. Il caresse, - n'est pas Il caresse avant de blesser - (L'amour avec le chat dessin qui est chez mon frère). Le mien est le pendant et représente deux enfants dont l'un chatouille l'autre un chien est couché près d'eux. Dessin estompé sur papier blanc $\mathrm{H}^{\mathrm{r}} 40 \mathrm{~L} .18$ acheté 800 $[F]$ à la vente Van Os peintre de fleurs.

265. Les quatre heures du jour. Dessin sur papier bleu rehaussé de blanc L. $15 \mathrm{H}^{\mathrm{r}}$ 6. Réunis dans le même cadre. Ces compositions ont été reproduites en camaïeu dans l'hôtel Lanois au-dessus des portes du salon où étaient représentées les figures allégoriques le plaisir la philosophie etc. transportées aujourd'hui à Ferrières.

356. Dix dessins. La poésie, l'étude, l'industrie, etc. pour la déco-

29. Petit profil de femme croqué à la plume en marge.

30. Souligné et accompagné de ce commentaire à l'encre noire en marge : " pas vrai ". 
ration de l'hôtel de ville lors du mariage de Napoléon avec Marie Louise. H. 36 L. 24. Je crois me rappeler sans certitude que cette série appartenait au peintre Trezel ${ }^{31}$ élève de Prud'hon. Mais ce que j'affirme c'est que mon père lui acheta 600 [F] Minerve conduisant le génie des arts à l'immortalité.

La plupart des académies qui se trouvent tant chez mon frère que chez moi viennent de la même source. Vers 1850 l'atelier de Trezel était rue [M.-]le[-]Prince[,] maison Jean Goujon. J'allais souvent le voir pour m'entretenir de Prud'hon avec lequel il avait travaillé. Il me montra une copie xe " copie " réduite de L'âme brisant ses liens; cette copie était de lui et retouchée par le maître. Elle fut achetée par [M.] Dromont comme originale. Mais c'est une erreur : 2 ou 3 touches savantes avaient trompé l'amateur.

Je ne puis rien affirmer cher Monsieur et prouver que le portrait de femme soit celui de [M.] de Polignac. Il fut apporté par Tusbry ${ }^{32}$, violon de l'opéra qui faisait le métier de brocanteur. Á l'appui de beaucoup de dissertations, il disait comme témoignage que la princesse étant gouvernante des enfants de France, l'artiste avait représenté son modèle appuyé sur le berceau du Dauphin ct que ce mcuble par sa richesse marquait son origine. La jeune femme que j'ai sous les yeux est blonde. Elle est coiffée d'un espèce de pouf surmonté de deux plumes blanches. Le corsage de la robe à raies bleues est en partie caché par un très long tissu blanc noué derrière la taille à la façon Marie-Antoinette. Mon frère m'a donné une gravure représentant l'amie de la Reine peu de temps avant sa mort. Je trouve une grande analogie entre les deux figures. Pas d'autre preuve.

Je suis prêt cher Monsieur à répondre à toutes vos demandes et s'il me survient des idées je vous les communiquerai. En attendant agréez l'expression de mon plus affectueux dévouement.

C. Marcille

Le dessin des vendanges a été acheté $770[\mathrm{~F}]$ à la vente Vignon architecte rue de l'arbre [illisible] Décembre 1846.

31. Nom peu lisible.

32. Nom peu lisible. 
Oisème 18 Juillet $1875^{33}$

[f. 7]

P. S. La Psyché était si mal encadrée qu'après la mort de mon père je l'ai portée chez Beugnet. La note qui était derrière a été détruite et je suis à me demander si ce n'est pas 3000 [F] qu'elle a été achetée chez Odiot; je crois que c'est 3100 . Mon frère vous le dira, sinon la personne qui a poussé ce dessin existe et pourra me renseigner. Après chaque vente mon père mettait derrière le dessin le prix de l'adjudication.

Les dessins pour la décoration de l'hôtel de ville ne viendraientils pas de chez Odiot? Veuillez voir dans le catalogue. Je n'affirme qu'avec certitude [que] :

- c'est le marquis Maison qui poussa la Psyché

- si vous désirez un croquis de [M.] de Polignac je vous l'enverrai.

33. Edmond prépare le Catalogue raisonné de Prud'hon, qui paraîtra en 1876. 
1. [f. 10]

\section{EUDOXE MARCILLE}

Chers Messieurs,

Je puis vous renouveler l'invitation que j'ai été si heureux de vous adresser hier. L'ami Duplessisxe «Duplessis (G.) » sera des nôtres. À Jeudi à 6 h. et _. Je serai enchanté de vous recevoir.

Agréez, chers Messieurs, l'assurance de mes sentiments respectueux et tous dévoués.

$13 \mathrm{X}^{\text {bre }}[18] 63$

Eud. Marcille

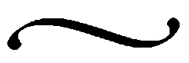

2. [f. $11-12]$

\section{Chers Messieurs,}

J'aurais désiré, toute la matinée, pouvoir vous remercier de m'avoir procuré l'honneur de connaître la Princesse Mathilde : mais j'ai eu du monde depuis onze heures et demi jusqu'à la nuit. Je regretterais, chers Messieurs, que la journée se passât sans vous dire combien j'ai été, et combien je suis sensible à votre bienveillance pour moi. Grâce à votre bonne recommandation, l'accueil de la Princesse a été, hier soir, excellent : dînant en ville, je n'ai pu aller rue de Courcelles qu'à onze heures, et j'en suis sorti après minuit heureux d'avoir entendu un mot aimable sur le compte de ma fille qui recevrait aussi une invitation, si elle avait quelques années de plus.

Croyez, chers messieurs, à mes sentiments affectueux et tout dévoués,

Paris, 18 Janvier [18] 64

Eud. Marcille

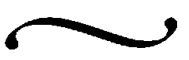

3. $[\text { f. } 13]^{34}$

La princesse m'a fait l'honneur de m'inviter à dîner ché̌ elle aujour-

34. Papier bleu plié en quarre. Message probablement laissé sur place. 
d'hui, chers Messieurs. Vous devant cette faveur, je suis venu pour vous serrer la main, et pour vous prévenir que je reviendrai à $6 \mathrm{~h}$. et _. Je serais heureux de vous accompagner rue de Courcelles, et $j$ 'espère qu'il vous sera possible d'occuper dans la voiture les deux places que je vous réserve.

Agréez, chers Messieurs, l'assurance de mes sentiments affectueux et dévoués

3 heures

Eud. Marcille

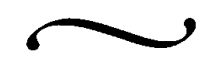

4. [f. 14-15]

\section{Chers Messieurs,}

Mon frère, ma belle-sœur et mes nièces arriveront à Paris samedi prochain. Soyez, je vous prie, assez bons pour venir dîner avec eux, de Jeudi en huit, le Jeudi 23, à 6 h. et _. J'espère, chers Messieurs, que vous serez libres ce jour-là. Je vous demande pardon d'avance du bruit qui sera fait autour de vous. Chacun à sa manière vous prouvera le plaisir que cause toujours votre présence rue d'Hauteville.

Recevez, chers Messieurs, l'assurance de mes sentiments affectueux et dévoués,

Paris 13 Avril 1868

Eud. Marcille

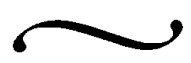

5. [f. 16-17]

Chers Messieurs,

J'irai vous voir, vous serrer la main et vous exprimer le regret de ne plus être votre voisin ${ }^{35}$. Avant ma visite, je vous prie de ne pas disposer de votre porte-cartons. Je serai heureux de le posséder et sa vue sera toujours pour moi une source de bons souvenirs.

35. Les Goncourt ont emménagé dans leur maison d'Aureuil. 
Veuillez agréer, chers Messieurs, l'assurance de mes meilleurs sentiments,

Paris, 8 Mars 1869

Eud. Marcille

6. [f. 18-19]

\section{Cher Monsieur,}

Le tableau que j'ai au Musée d'Orléans est charmant ${ }^{36}$ : il est gravé sous le nom de Watteau, il représente le singe sculpteur qui fait pendant, je crois, au singe peintre. Vous trouverez la gravure dans l'œuvre de Watteau, et plus tard, je pourrai vous envoyer la copie xe "copie" de ma fiche ${ }^{37}$.

Vous n'attribuez pas à Watteau, cher Monsieur, un dessin que mon frère croyait être de ce maître; il représente un jeune homme debout jouant de la flûte près d'une jeune fille qui a le bras gauche appuyé sur une cage. J'ai un autre dessin de l'auteur du Voyage à Cythère: c'est un croquis d'après un fragment de l'esquisse de Rubens qui m’appartient et qui est à l'Exposition des Alsaciens-Lorrains. Mais partant pour la guerre; de plus une peinture, des fleurs sur une musette.

Le 5 Juillet, cher Monsieur, l'Exposition des œuvres de Prud'hon sera terminée ${ }^{38}$. Avant de rendre les tableaux et dessins aux personnes qui me les ont confiés, je demande l'autorisation de les faire photographier. M. Braun est chargé de cette entreprise. Voulez-vous permettre que votre dessin soit photographié?

Agréez, je vous prie, cher Monsieur, l'expression de mes meilleurs sentiments,

Eud. Marcille

Paris, 27 Juin 1874

36. Eudoxe Marcille est directeur du Musée d'Orléans depuis avril 1870.

37. Edmond prépare le Catalogue raisonné de Watteau, qui paraîtra en 1875.

38. Exposition des dessins de Prud'hon, organiséc à l'École des Beaux-Arts à l'initiative d'Eudoxe er Camille Marcille, pour venir en aide à la fille de Prud'hon (voir Georges Duplessis, "Les cxuvres

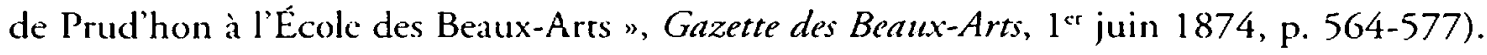


7. [f. 20-21]

Cher Monsieur,

Je ne puis, à mon grand regret, aller à Auteuil, étant toujours très occupé à Paris. Si vous êtes pressé pour votre catalogue de l'œuvre de Watteau, je désire vous prévenir, avant votre départ, que le tableau du Musée d'Orléans - Le Singe sculpteur - est peint sur toile, et que la gravure est de la grandeur du tableau.

A la fin du mois, quand j'aurai rendu tous les tableaux et dessins qui m’ont été confiés, je pourrais vous donner des renseignements plus précis.

Soyez sans inquiétude pour votre dessin : je le garderai chez moi. Je vous remercie de nouveau de me l'avoir confié.

Croyez, cher Monsieur, à mes meilleurs sentiments,

Eud. Marcille

Paris, 4 Juillet 1874

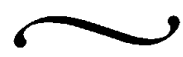

8. [f. 22-23]

\section{Cher Monsieur,}

Je prends la liberté de réclamer de vous un mot pour m'autoriser à publier dans mon catalogue du Musée d'Orléans la notice d'un Roguet qui a paru dans le journal l'Éclair ${ }^{39}$. Si vous voulez bien m'accorder l'autorisation demandée, dites-moi, je vous prie, quelle est la date du journal.

Votre excellente notice, cher Monsieur, m’a été communiquée par $\mathrm{M}$. Roguet, frère de l'artiste, directeur des contributions directes à Tarbes. J'ignorais que vous eussiez connu si intimement l'éminent artiste dont le Musée d'Orléans possède presque toutes les œuvres.

39. Catalogue des tableaux, statues et dessins exposés au Musée d'Orléans, Orléans, H. Herluison libraire-éditeur, 1876, in-12, 324 p., sans nom d'auteur. La notice sur Roguet figure p. 220-227, et l'article des Goncourt est cité p. 225-227. Le sculpteur Louis Roguet, né à Orléans en 1824, est grand prix de Rome en 1849. Déjà malade au moment où il passe le concours, il meurr à Rome en 1850 . 
J'ai beaucoup entendu parler ici de Roguet : tout ce que vous avez écrit sur lui résume on ne peut mieux les éloges qui m’en ont été faits.

Je suis ici depuis le $127^{\text {bre }}$ : j'ai consacré tous mes instants à améliorer le Musée. Vers le 15, j'irai voir mon frère et ma belle-sœur. Je ne serai à Paris qu'à la fin du mois. Un Vendredi, dans le courant de Janvier, je vous porterai votre dessin de Prud'hon.

Ma fille se porte bien. Je suis heureux de penser qu'au mois de février, elle deviendra mère ${ }^{40}$.

Agréez, je vous prie, cher Monsieur, l'expression de mes meilleurs sentiments

Eud. Marcille

au Musée

Orléans, $7 \mathrm{X}^{\text {bre }} 1874$

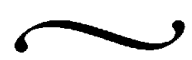

9. [f. 24-25]

Cher Monsieur,

Vous aimiez beaucoup votre frère : j'aimais beaucoup le mien. Les deux jeunes sont partis avant leurs aînés.

Hier, j'ai reçu une dépêche télégraphique à la campagne près d'Orléans, m'annonçant que mon frère était malade. Ma belle-sœur me disait de prendre le premier train pour venir auprès d'elle. Quand j'arrivai à Chartres, j'appris que mon bien aimé Camille avait été foudroyé, en quelques heures, par une congestion cérébrale : il est mort, le mardi 3 , à quatre heures du matin : il était né à Chartres, le $1\left[{ }^{[\mathrm{r}}\right]$ Mai 1816.

Je fais, cher Monsieur, une perte que vous comprendrez mieux que qui que ce soit. Mon frère était un homme supérieur : doué d'un sens droit, d'un esprit délicat, il avait voué sa vie au culte du beau dans les lettres et dans les arts. Oisème était, jadis, un séjour enchanteur pour moi : aujourd'hui, le silence de la mort y règne, et je ne

40. Marie Marcille s'est mariće le 29 janvier 1872; Edmond assistait à la cérémonis (Journal, 29 janvier 1872). 
dois penser qu'à aimer, de plus en plus, mes nièces qui, comme leur mère, ont si bien apprécié le mérite de celui qu'elle pleure [sic].

Je vous serre, cher Monsieur, affectueusement la main, persuadé que vous n'oublierez pas mon cher Camille.

Eud. Marcille

Oisème, 4 Août 1875

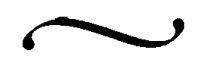

10. [f. 26-27] ${ }^{41}$

Cher Monsieur,

J'ai dû passer quatre jours loin de Paris pour assister, à Orléans, à l'inauguration des expositions qui y ont lieu à l'occasion du Concours régional. Je n'ai pu m'occuper de ces expositions, étant retenu à Paris pour le travail qui précède ce qui suit l'ouverture du Salon.

De retour, depuis hier, je cherche à vous faire plaisir, en vous communiquant les renseignements que vous me demandez. Voici, cher Monsieur, les prix que vous désirez connaître.

$\begin{array}{cl}\text { Nos } 97 & 3500 \\ 98 & \} 315 \\ 98[99] & \\ 100 & 9000 \\ 101 & \} 1255 \\ 102 & \\ 103 & \} 1200 \\ 104 & \end{array}$

Lundi, je serai chez moi, heureux de vous y recevoir et bien disposé à vous être agréable. Je vous remercie de tout ce que vous faites pour la mémoire de notre cher Prud'hon.

Agréez, je vous prie, cher Monsieur, l'expression de mes meilleurs sentiments,

Paris, 11 Mai 1876

Eud. Marcille

41. Lettre entourée de noir. 


\section{MADAME CAMILLE MARCILLE}

\section{1. [f. 28]}

En vérité Juliette a bien raison de vous aimer de plus en plus. Vous gâtez les enfants et les parents, mon cher Monsieur, et Oisème luimême, devient supportable quand on y vit entre vos livres et vos joujoux ${ }^{42}$.

Je supplie votre grand frère $e^{43}$ de nous donner quelques jours au printemps. Nous lirons, nous causerons, nous ferons quelques excursions. Enfin nous serons si heureux de vous voir que vous viendrez pour ce seul motif : et nous fixerons alors l'époque de la réunion d'automne avec les cousins.

Pour moi, si je retourne à Paris je veux aller voir vos merveilles au grand jour, car ma visite chez vous ne m'a presque laissé que des regrets ${ }^{44}$.

Mille bons et affectueux souvenirs, cher Monsieur, de la part des parents. Les six enfants vous accablent de tendresses.

Walckenard Marcillexe "Marcille (C. et E.) " 45

Oisème 6 Janv. [18]64

On garde votre lettre dans les archives de la famille. Juliette voulait la faire encadrer!

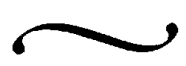

2. [f. 30-31]

Mon gendre, je voudrais vous faire relire Les Maîtresses de Louis $X V$. Car je vous plains, vous autres auteurs, vous n'avez pas le plai-

42. Voir la lettre de Jules à Juliette Marcille, citée dans les Lettres publiées par Edmond en 1885 (p. 222-223 dans l'édicion Flammarion-Fasquelle de 1930), qui accompagne l'envoi d'une caisse de jouets. Elle est datée du $1^{\text {rr }}$ janvier 1863, mais la correspondance de Madame Marcille et de ses

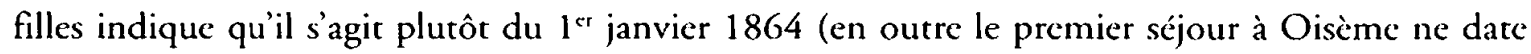
que d'octobre 1863).

43. Souligné.

44. I.e Journal évoque un sćjour à Paris de la famille de Camille Marcille à la date 17 décembre 1863.

45. $\mathrm{M}^{\mathrm{mcc}}$ Marcille est la fille de l'érudit Charles-Athanase Walckenacr, selon l'orthographe usuclle. 
sir de lire vos ouvrages, une première fois, tout d'un trait : vous n'avez pas le charme des découvertes et de l'imprévu. Enfin vous êtes juges et partie. Le public est bien plus heureux! et pour être un public de campagne je n'en ai pas moins apprécié toutes les qualités sérieuses et charmantes de cet aimable livre, et cela depuis la préface jusqu'à la dernière page. Oui, vraiment j'ai été ravie, et je suis en colère contre Sainte-Beuvexe "Sainte-Beuve (C. A.) ». Voilà mon avis.

Vos petites amies ont été bien contentes de vous aussi. Si vous aviez vu cette joie, ces cris, ces bonheurs pour toutes vos lettres vous ne regretteriez pas le temps que vous avez volé au grand frère. Eudoxe me trouve bien favorisée de posséder un autographe signé E. de Goncourt! Je crois qu'un peu plus il me l'aurait volé. Mais je fais bonne garde! Et la lettre serait signée Edmond tout court que j’y tiendrais tout autant. Voyez comme je n'ai pas l'amour de l'art! et comme je ferais pitié aux collectionneurs! Notre pauvre Marie a été toute souffrante : elle s'est mise au lit le jour de votre départ et enfin elle a eu une petite fièvre bilieuse qui ne l'a quittée qu'avant-hier. La voilà tout à fait bien et elle s'en va aujourd'hui s'établir chez la tante Letellier à Long-Saulx : c'est encore un départ et certes le père et la fille méritent d'être regrettés. Aussi les jours vont pour moi au rebours de l'almanach, allongent au lieu de diminuer, et Oisème va me paraître une vraie campagne. Or vous savez comme je suis champêtre!

Adieu mon gendre; vous avez une belle-mère par trop bavarde; c'est là son moindre défaut! Vous n'aurez en compensation que la solidité de son cœur et de ses sentiments pour vous.

Walckenard Marcille

Camille me charge pour vous deux de ses meilleurs souvenirs et de ses remerciements pour votre aimable lettre.

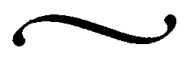

3. [f. 32-34]

Cher Monsieur,

Nous avons au moins le mérite de vous avoir appréciés et de bien vous regretter.

Ces quatre petites folles vous disent, en riant, la vraie vérité et je crois qu'elles restent encore au[-]dessous, en ayant l'air de la dépas$\operatorname{ser}^{46}$ : car Oisème n'est plus Oisème depuis que vous l'avez quitté et 
je vous assure que d'autres pourront y venir sans jamais vous remplacer. Nos enfants parlent de vous à toute heure, à tout propos; elles se répètent tous les bons mots de [M.] Jules et ma Jésus ${ }^{47}$ a pour vous des éclairs de souvenirs et de tendresse qui la font trouver adorable.

Vos portraits nous font encore plaisir à voir ${ }^{48}$. Mais on n'est jamais entièrement content des portraits de ceux qu'on aime. L'âme n'y est pas, on n'a que la figure.

Nous avions tous le cour gros en nous quittant, n'est-ce pas? et il me semble que le mien en est resté au moment du départ.

Je vous recommande la santé de mon gendre et je lui recommande la vôtre. Il faudra que ce gendre se mette quelque fois en classe pour nous écrire : je l'en aimerai davantage, si toutefois on peut aimer un gendre plus que je fais.

Adieu, cher Monsieur, on vous aime et on vous regrette tant ici que je crois que vous avez le droit d'être ingrats. [1865]

Cécile

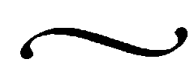

4. [f. 34]

Je crois que je ne vais vous être absolument bonne à rien pour les renseignements que vous me demandez. Vous n'imaginez pas comme mon intelligence est fermée par certains côtés, et comme elle est lente à comprendre. Il faudrait que je puisse passer une heure avec vous et que vous me missiez au courant de toute votre histoire. Alors je crois que les idées me viendraient, puisqu'il s'agit d'un sentiment vrai ; ma sœur fournirait à ma mémoire des traits d'exaltation et d'obstination religieuse; et la Jésus est tout un poème de sensibilité et d'adoration

46. La lettre de $\mathrm{M}^{\mathrm{me}}$ Marcille était probablement jointe à celle de ses filles, numérotée f. 66-68, et datée du 5 juillet 1865. Le Journal évoque un séjour des Goncourt à Oisème à la date du 27 juin 1865.

47. La petite Jane, la plus jeune des filles de C. Marcille.

48. Probablement peints par C. Marcille au cours du séjour des Goncourt. Le Jotirnal (24 juillet 1879) évoque un portrait d'Edmond par C. Marcille. 
filiale dans lequel nous pourrions puiser ${ }^{49}$. Mais, je vous le répète, il faudrait pour cela que nous puissions causer bien tranquillement et être nous-mêmes. C'est si bon de pouvoir être soi-même tout bonnement et tout bêtement! - mais les femmes de ménage ne croient plus à la simplicité : la vôtre serait scandalisée, j'en suis sûre, que j'allasse passer deux heures avec vous pour lire votre ouvrage; mon cher Monsieur Edmond, il ne nous sert à rien de vieillir, à nous autres femmes! La chose est pourtant si dure que nous devrions avoir des compensations.

Adieu, et j'espère au revoir. Je vous écris si à la hâte que j'oublie très probablement le français et même l'orthographe. Mais notre amitié ne se trouve pas dans les dictionnaires. Comptez bien tous deux sur la mienne, bonne, franche et sincère

C. Walckenard Marcille

Envoyez-nous votre livre quand il sera fini; je le lirai avec plus de plaisir en le tenant de vous (et surtout ne gardez pas rancune à ma bêtise de ce qu'elle ne sait pas avoir une idée à votre service).

5. [f. 36-37]

2.3 juin au soir [1870]

Mon pauvre et cher ami,

Comment ne vous ai-je pas vu dans ce moment où mon cœur est si déchiré, si pénétré de votre douleur ${ }^{50}$ ? Comment ne m'a-t-il pas été donné de mêler mes larmes aux vôtres; de vous dire tout ce que mon cœur a souffert pour vous et avec vous depuis six mois! Peutêtre l'avez-vous deviné, car il est des affections dont on ne doute pas, même quand elles semblent rester muettes; si vous avez pressenti que mon amitié et mes pensées étaient toutes à cette chère et triste maison d'Auteuil, je vous en remercie de toute mon âme.

J'ai frappé bien longtemps aujourd'hui à la porte de cette maison.

49. Les Goncourt sont probablement à la recherche de documents complémentaires pour Madame Gervaisais, qui paraîtra en 1869.

50. Jules est décédé le 20 juin 1870. 
Mais elle m'est restée fermée comme l'est maintenant pour vous la tombe de votre frère chéri.

Ah! Mon ami, je me sens bien malade et bien épuisée, je ne vous verrai plus sans doute; mais sachez, sachez bien que partout où vous serez, et quel que soit le temps que nous ayons à vivre l'un ou l'autre, il y a un cœur qui vous est et vous sera toujours entièrement uni dans le souvenir de cet être si bon, si charmant, si digne d'être votre frère!

Adieu, il me semble que mon cœur s'apaise un peu à la pensée que vous connaîtrez toute l'étendue de mon affection et de mon attachement. Mais qui pourra apaiser le vôtre, mon pauvre ami! Votre isolement et votre douleur m'épouvantent!

C. W. Marcille

6. [f. 38-40]

Combien je vous remercie mon cher ami - je ne peux plus vous appeler autrement depuis que vous êtes malheureux - de m'avoir répondu, de m'avoir parlé de notre pauvre cher Jules! et des impressions de votre cœur. Il me semble que je vous comprends bien en toutes choses; et que si nous pouvions plus souvent échanger nos idées, celles surtout qui viennent du cœur, je pourrais adoucir un peu l'amertume et le déchirement de vos souvenirs. Mais il n'y faut pas penser. Je n'ai dit à personne $e^{52}$ que je vous avais écrit, ni que vous m'avez répondu ${ }^{53}$; cela vous étonne, n'est-ce pas! Vous vous demandez comment une chose si simple, si honnête, qui est si naturellement l'expression d'une amitié fraternelle et pure, peut avoir besoin d'être cachée. Mais il en est ainsi, je n'en murmure pas, quoique j'en sois affligée. Je sais bien que les gens les plus excellents, et du meilleur

51. Cetre lettre semble précisément évoquée dans le Journal (30 juin 1870). L'erreur de date est probablement le fait d'Edmond, qui rédigera rétrospectivement les entrées du Journal consécutives à la mort de Jules.

52. Les expressions en italiques sont soulignées dans la lettre.

53. Voir Journal, 23 avril 1868 : M"me Marcille dit à Edmond qu'ils ne se verront plus qu'une fois par an, à cause de la jalousie de son mari. 
esprit, ont parfois des faiblesses - je dirais presque des ridicules qu'il faut leur pardonner, quand ils émanent d'un excès de tendresse. La sympathie de [M.] M. pour vous est réelle et sincère. Mais pour qu'elle subsiste ainsi, il faut que je ne semble pas trop la partager. Vous me dites que je ne suis pas si malade que je le crois, et que je vous reverrai. Ah! que je le souhaite, mon cher ami! Car une heure de causerie nous ferait du bien à tous deux et je la désire depuis longtemps. D'ici là nous avons ce beau et grand rendez-vous de la Providence pour nous retrouver. Vous avez deviné que mon cœur s'élevait souvent vers elle depuis votre malheur. Oui, c'est là que je me réfugie. C'est là que j'unis ma pensée à ceux que j'aime, à ceux qui ne sont plus. Ce grand tout du Bon Dieu, dégagé de toutes les petitesses dont on l'entoure souvent, est le vrai refuge des âmes qui souffrent, qui espèrent ou qui regrettent. C'est à lui seul que je vous confie et que je confie mes chers enfants adorés, quand la pensée me vient que je dois les quitter bientôt. Savez-vous, mon pauvre ami, ce qui fait que nous nous sommes compris si vite? c'est que nous avons bien compris tous deux le vrai amour maternel : oui, vous avez aimé votre Jules comme j'aime ma Jésus : et cet amour maternel c'est le seul vrai et grand amour. Il est si désintéressé qu'il doit ressembler un peu à celui que la Providence a pour ses enfants. Il faut y croire, mon ami, à cette Providence, quoiqu'elle vous ait laissé devenir malheureux. Car nos enfants nous trouvent injustes quelques fois [sic], malgré notre amour pour eux; et nous, dont l'esprit est si borné, nous murmurons aussi, parce que nous ne comprenons pas. Mais notre instinct et notre cœur nous poussent vers Dieu au moment même où nous murmurons contre lui.

J'aurais voulu aller sur la tombe de notre pauvre Jules pour bien penser à vous et à lui. Le désir était si grand que le sacrifice est grand de ne pouvoir le faire. - Car je ne le peux pas. - Non, même après la mort, il ne faut pas que je semble trop dévouée à mes amis. Que je suis heureuse au moins, d'avoir gardé toutes les lettres tous les moindres souvenirs de nos chers Goncourt. Il y a des enfantillages de Jules que j'ai encore. Il y a jusqu'à une feuille de rose, la dernière qui ait été cueillie avant votre départ de Oisème, que j'ai gardée, comme un charmant souvenir d'un bon temps qui ne reviendra jamais. - Jamais! ah! que ce mot est dur n'est-ce pas, et qu'il est cruel pour vous en ce moment! - Je vous envoie ma pensée de ce temps-là. 
Mettez-la dans un livre qui ait appartenu à votre cher enfant. Voulezvous, mon ami, envoyer (le plus tôt possible) une carte à vous, avec un mot affectueux pour [M.] M. et pour moi, comme celle que vous avez envoyée à Eudoxe. Je vous demande ce service. Adressez cette carte rue d'Hauteville. Nous ne retournerons à Oisème que dans quelques jours.

Au revoir, n'est-ce pas? Je ne veux pas encore vous dire adieu. Et si ce revoir n'est pas ici[-lbas, qu'il ait lieu chaque jour dans notre cœur, auprès de Dieu, dans le souvenir chéri de notre Jules ${ }^{54}$.

C. W. Marcille

7. [f. 41]

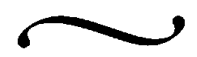

Cher Monsieur,

Mardi 6 février

Nous serons chez vous jeudi à deux heures. J'ai fait hier un pèlerinage qui m’a fait du bien au cœur. Et après ma visite à Auteuil, je retournerai dans ma solitude avec de doux souvenirs.

Votre vieille et vraie amie C. W. Marcille

Si vous vous ne pouvez pas être chez vous jeudi, prévenez-moi.

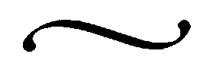

8. [f. 42-43]

Cette vie agitée (et bête) que l'on mène ici quand on y est en passant, m’a épuisée et je vais rester forcément chez moi aujourd'hui.

54. Voir aussi Journal, 4 février 1872 : "Je la trouve dans son salon, où il fait presque nuit et où la chaleur est écœeurante. Elle est vêtue d'une espèce de deuil violet, dans lequel l'élégance de sa personne a une grâce sévère, une grâce triste. Près d'elle, une vieille femme sourde cherche à deviner, sur ses lèvres, les paroles qu'clle me dit. Elle me parle de sa mort prochaine, qui ne fera pas de vide. Son mari est excellent, mais il se consolera avec la pcinture. Elle ne désire qu'une chose : marier Juliette, sa fille aînée, qui se chargera de Jeanne, sa chéric. Alors elle sera prête à mourir... sans regretter grand-chose. I À la fin, elle me demande la place de la tombe de mon frère, pour y aller en cacherte, un jour qu'clle aura beaucoup de visites à faire. " 
Jusqu'à 9 heures pourtant, car je ne veux pas priver mes filles d'un plaisir qu'elles se promettent ce soir. Demain, depuis deux heures, j'espère pouvoir me reposer encore. Puis, si vous ne pouvez venir chez moi ces deux jours-ci, je tâcherai d'être rentrée toute la semaine prochaine à 4 heures _ et enfin d'aller vous voir avant mon départ, qui approche.

Mais ni chez vous ni chez moi nous ne pourrons causer doucement et à l'aise de nos chers souvenirs, qui restent pour moi comme unc relique du passé. Donnez-moi pourtant ce bonheur de vous serrer la main et tentez, comme par hasard, de me trouver ici. Il a fallu pour moi toute la délicatesse du devoir pour que je n'aie pas encore été voir cette chère maison où notre cher Jules a vécu - et cette autre où il repose.

Adieu - et au revoir encore une fois j'espère.

Samedi matin

W. M.

Samedi matin

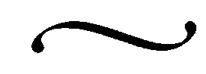

9. [f. 44-45]

Mardi soir

Cher monsieur et ami,

Ma petite Jane étant un peu souffrante je resterai demain toute la journée chez moi -13 rue St[-]Lazare. Si vous passez près d'ici, venez frapper à ma porte : je serai si heureuse de vous voir. Ne venez pas après dîner. Je serai entourée de monde à cette heure-là et je crains les foules. Je ne sais pas encore quand je pourrai aller vous voir, car je suis entourée d'embarras de toute sorte; et je dois attendre Eudoxe à qui j'ai demandé de m'accompagner. Mais aussitôt que ce sera possible je vous écrirai pour vous prévenir de notre visite. Pauvre ami! que je voudrais mettre un peu de ouate autour de votre cœur! je sens que c'est impossible et mon cœur se serre en pensant à vous.

W. M.

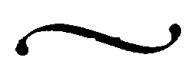


10. [f. 46-47]

Le Paraclet 8 juin [1873]

J'ai passé six semaines à Paris sans vous voir, cher Monsieur et ami. Le cœur tout plein de regret j'ai lu, en arrivant ici, la pièce que vous nous avez adressée ${ }^{55}: j^{\prime} y$ ai reconnu toutes les spirituelles saillies de notre cher Jules (il me semblait encore l'entendre!) et toutes les pensées qui viennent de vous. Je ne sais ce qu'elle serait à la scène, mais la lecture de cette pièce m'a plu infiniment. Je la trouve supérieure à $H$. Maréchal, et vraiment digne de vous deux. Merci de ce souvenir et du mot affectueux que vous avez écrit sur votre carte. Vous n'imaginez pas ce qu'il m'en a coûté de ne pas revoir votre maison et les fleurs de votre jardin! Vous savez bien au moins qu'il y a pour moi un triste et pieux rendez-vous auquel je n'ai pas encore manqué; auquel je ne manquerai jamais. Vous pouvez tous les deux compter sur mon cœur, comme je compte sur votre amitié et sur votre souvenir.

C. W. Marcille

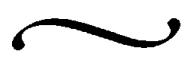

11. [f. 48]

Cher Monsieur et ami,

Je veux que vous appreniez par moi, un des premiers, le mariage de ma fille Juliette. Elle épouse un Capitaine des Dragons, brave et loyal garçon, dont l'intelligence nous donne autant de garanties pour sa carrière militaire, que les qualités de son cœur nous en donnent pour le bonheur de notre fille.

Vous vous associerez, non pas à ma joie, (une mère n'en éprouve guère en une telle circonstance) mais à tous les sentiments qui agitent notre cœur en ce moment. Vous savez qu'il y a bien longtemps

55. La l'atrie en danger, écrite en commun avec Jules, paraît en 1873. Les deux frères achèvent de rédiger la pièce le 11 octobre 1867 (voir Journal); ils lui donnent alors le nom de Blanche de La Rochedragon. Sous le titre de Mademoiselle de La Rochedragon, clle sera refuséc par le comité de lecure du Théâtre-Français le 7 mars 1868. 
que je compte sur votre amitié et que la mienne vous est acquise bien profondément et à toujours.

Walckenard Marcille

Oisème 28 [sic] [1873 ou 1874$]^{56}$

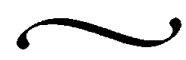

12. [f. 50]

[1873 ou 1874]

Cher Monsieur et ami,

Ce n'est pas un simple billet d'invitation qui doit vous demander d'assister au mariage de ma fille. C'est moi qui vous en prie, comme de la meilleure marque d'amitié que vous puissiez donner à vos amis de Oisème. Je ne pourrai pourtant pas vous y recevoir après la cérémonie. Mais nous nous réunirons, à Chartres, dans une maison amie, qui sera la mienne ce jour-là.

Les trains quittant Paris sont à 7 h 30 et 8 h du matin. Mon frère se trouvera à la gare de Chartres pour indiquer aux arrivants l'hôtel de France, où ceux qui seront partis à jeun pourront déjeuner.

Si vos affaires ou votre santé vous empêchaient, malgré mon désir, de répondre à mon appel, je vous demande de penser à votre vieille amie le 9 mars, car ce jour sera bien pénible pour elle.

Votre bien dévouée Walckenard Marcille

13. $[\text { f. } 52-53]^{57}$

Cher Monsieur et ami,

Mon beau-frère me dit que vous avez manifesté le désir de coopérer au succès de la vente de nos tableaux ${ }^{58}$. Nous vous

56. Voir, pour la datation, la note à propos de la lettre de Camille Marcille [f. 5-6].

57. Lettre entourée de noir.

58. La vente de Camille Marcille a lieu du 6 au 9 mars 1876. Le catalogue (Catalogue des tableaux et des dessins formant la collection de feu $M$. Camille Marcille) est préfacé par Paul de Saint-Victor. 
sommes, tous deux, profondément reconnaissants de cette pensée.

Le secours de votre amitié et de votre talent est le plus efficace que nous puissions avoir; et, non seulement je l'accepte, mais je le sollicite. La dispersion de tous ces objets, précieux pour nous à tant de titres, m'est bien dure. Vous le devinez, j'en suis sûre, quoique vous n'ayez pas eu à subir la même épreuve. Mais votre propre douleur vous rend capable de comprendre toutes les autres.

Mes filles et moi vous renouvelons l'expression de notre entière sympathie et de notre affectueux attachement.

Walckenard Marcille

Oisème 8 février [1876]

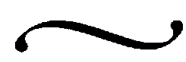

14. [f. 54-55]

Cher Monsieur et ami,

Je suis si confiante dans l'intérêt que vous nous portez et dans les chers souvenirs qui affermissent encore notre amitié que je n'ai pas douté de votre concours. Vous avez employé, j'en suis sûre, toute la chaleur de votre excellent cœur et tout le charme dc votre esprit pour contribuer au succès de cette vente, et vous avez réussi ; car Eudoxe nous assure que ce succès est réel; et personne ne peut être meilleur juge de notre cause que ce frère vraiment incomparable dont le dévouement et le zèle font notre admiration et nous inspirent les sentiments de la plus vive et la plus profonde reconnaissance.

Pour moi, en comparant le résultat au sacrifice, je dois toujours le trouver incomplet. Il ne reste plus rien à Oisème, mon cher ami. Celui qui m'était la vie, ces tableaux qui en faisaient le charme, tout a disparu. Je ne puis vous dire quel vide s'est fait dans mon cœur, dans ma vie, dans ma maison : les jours se succèdent sans but et sans projets; et la pensée du devoir accompli est la seule qui puisse me donner du courage.

Vous souffrez aussi et vous me comprenez. Comme je vous plains mieux depuis que je suis malheureuse : et pourtant je devinais bien avant que vous deviez beaucoup souffrir. 
Je vous serre la main bien affectueusement et je vous assure encore de mon ancienne et inaltérable amitié.

Oisème 12 mars [1876 ]

Walckenard Marcille

15. [f. 56-57]

Je voudrais, cher Monsieur, que vous n'apprissiez pas par un tiers le mariage de ma fille Marguerite. Elle épousera dans un mois un homme bon, intelligent et distingué, qui la récompensera, je l'espère, des sacrifices et du dévouement qui lui sont imposés, depuis trop longtemps déjà, envers sa sœur et envers moi.

Vous aurez sans doute appris, par mes neveux, ou par mon beaufrère, que depuis deux ans ma pauvre Jane est bien malade; cette enfant que vous avez vue si vigoureuse n'a plus qu'un souffle de vie.

Nous allons, d'étapes en étapes, cherchant un remède à ce mal inexplicable; et ne trouvant pas même un soulagement à ses souffrances.

Je viens, encore une fois, (peut-être une dernière fois!) de réunir mes enfants dans cette retraite de Oisème pour moi si pleine de chers souvenirs. C'est là que je pense à vous écrire; et où il me semble vous voir encore avec ce bien aimé frère que nous avons regretté ensemble.

Je suis sûre, cher Monsieur et ami, de ne pas réveiller en vains ces douloureux, mais touchants souvenirs d'une vieille et sincère amitié; et je suis sûre aussi de trouver dans votre cœur un écho sympathique pour le rayon de bonheur qui m'arrive à travers tant de tristesses.

Oisème lundi soir

Walckenard Marcille

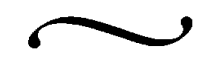

16. [f. 58]

Avant de commencer la lecture de votre livre qui aura, à tous les titres, un si puissant intérêt pour moi ${ }^{59}$, je veux vous remercier de ce constant souvenir. Vous le gardez à une vieille amie pour laquelle la

59. Probablement Les Frères Zemganno (1879). 
mémoire du passé vous répond de l'attachement présent - et jusqu'à la fin.

Walckenard Marcille

16 avril [1879]

17. [f. 59]

9 juin $[18] 84$

Mon cher ami,

Les lettres de votre frère sont des reliques aussi précieuses pour moi que pour mes enfants. Elles restent à Oisème dans le coffre où j'ai renfermé mes plus chers souvenirs. Si mon jardinier est assez intelligent pour les trouver, vous les aurez bientôt. Sinon, il vous faudra attendre mon retour là-bas, qui n'aura lieu qu'au commencement de juillet. D’ici à huit jours je pourrai vous donner une réponse définitive.

Notre pensée, à tous deux, retourne sans cesse en arrière : c'est un grand lien entre nous, et notre amitié est comme les roses de Oisème qui continuent à fleurir, toujours vivaces, pour les morts comme pour les vivants.

Je vous serre la main de tout mon cour ${ }^{60}$.

Walckenard Marcille

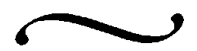

18. [f. 60]

Oisème 9 juillet [18]84

Mon cher ami,

Je suis bien émue en remuant ces papiers, en relisant ces lettres. Elles n'évoquent pourtant pas des souvenirs effacés ou disparus. Tout m'est présent; tout m'est vivace.

60. Edmond commente ainsi cette lettre le 15 juin 1884 dans le Journal: " Les femmes ont un art particulier, dans des phrases qui ne semblent rien dirc, de sous-entendre de délicates choses de cơur. J'ai reçu, ces jours-ci, une lettre tendrement charmante de $\mathrm{M}^{\text {mix }}$ Camille Marcille, à propos de la communication de lettres de mon frère que je lui demandais." 
Je ne crois plus avoir beaucoup de temps à aimer ceux que j'ai aimés et que j'aime; mais ce sera jusqu'au dernier jour.

Prenez de ces lettres ce que vous voudrez. Puis, rendez-les moi. Rendez-les moi toutes. Elles seront précieuses à mes enfants autant qu'elles me le sont à moi-même.

Si vous voulez que nous parlions encore une fois ensemble de notre cher Jules, de mon vénéré Camille, des temps heureux, venez passer, cet été, une journée à Oisème. Si vous deviez en éprouver une douce ćmotion, nous la partagerions de tout cœur avec vous.

W. M.

19. [f. 61]

Oisème 31 août [1884]

Mon cher ami,

J'aurais aimé à ce que vous revissiez notre Oisème pendant qu'il était encore éclairé par le soleil d'été; et, en me disant le motif qui nous prive de votre visite, vous doublez mes regrets.

Moi aussi je suis bien souffrante, je ne vis plus que d'une manière factice qui me fait regarder chaque année, presque chaque jour, comme une aumône. Je ne suis pas philosophe, je n'ai jamais su l'être; et cette prévision de la fin mattriste.

Gardez-moi jusque là et au-delà un bon souvenir. Je conserve celui de nos joyeuses réunions et de notre cordiale amitié comme un des meilleurs de ma vie.

Walckenard Marcille 
20. [f. 62]

Mon cher ami,

Jeudi soir $[1885]^{61}$

Nous revoyons Jules en relisant ses lettres; nous retrouvons avec lui les jours de bonheur où nous avions tous le cœur content, l'avenir devant nous. Le souvenir du passé, des êtres chers disparus est pour vous et pour moi une seconde vie, triste, mais encore douce puisque rien ne s'est effacé dans nos cœurs. Le mien vous conserve la vraie amitié des bons jours; et le témoignage que vous me donnez de la vôtre m'est bien précieux.

Walckenard Marcille

61. Les Lettres de Jules paraissent chez Charpentier en 1885. M $^{m c}$ Marcille remercie probablement Edmond de l'envoi du volume.

C'est la dernière lettre de $\mathrm{M}^{\mathrm{mc}}$ Marcille dans la Correspondance adressée aux Goncourt. Le Journal conserve cependant une trace de son passage à Auteuil le 21 juin 1885 : " Hier, j'ai eu une surprise - une surprise de cœur. Sur la tombe de mon frère, sur cette tombe uù il n'y a jamais que les couronnes apportées par moi, j'ai trouvé deux bouquets de goût, deux bouquets de roses et d'oillets entourés de petits panaches inconnus, une poussière de fleurs. Il n'y a que $\mathrm{M}^{\text {mc }}$ Camille Marcille capable de cette attention." C'est la dernière mention du personnage dans le Journal. 


\section{MESDEMOISELLES MARCILLE}

1. [f. $63-64]^{62}$

Monsieur,

[1864]

(moi je trouve que c'est bien sec;) mais maman trouve quc de dire mon cher ami ça n'est pas convenable, je vous aime de plus en plus. Vous m'avez écrit une si belle lettre que je veux la garder toujours. Je ne suis pas près de pouvoir en faire autant, car nous ne faisons que jouer du matin au soir et la nuit nous dormons naturellement. Votre grand frère qui est si frileux aurait bien froid ici. Le bassin est tout gelé. Que je voudrais donc que vous soyez là! Nous ferions des glissades ensemble et je vous tirerais par votre habit pour vous faire tomber : ça serait bien drôle. Mais je ne vous parle pas de vos jolis joujoux ${ }^{63}$. J'ai tant à vous dire que je ne sais par où commencer; tout était ravissant, je ne croyais pas que des garçons se connaissent si bien en joujoux; Jane a pris le petit lapin et moi tous les meubles; le secrétaire a été pour Marguerite il est bien joli. Quand vous viendrez je vous donnerai du thé dans mes tasses, enfin je suis enchantée et mes sœurs aussi et je voudrais bien vous voir, car je vous aime beaucoup; mais si j'ai été froide à Paris il ne il ne faut pas pas [sic] vous tourmenter c'est que nous étions pressées et qu'il ne faisait pas clair $^{64}$, je vous embrasse de tout mon cœur dites à votre grand frère qu'il vous donne bien à manger et surtout qu'il ne vous fasse pas avoir froid; mes cousins partent demain et je vais vous faire un essuie[]plume[s] pour que m'écriviez encore $\left[\right.$ sic ${ }^{65}$. Je vous envoie une

62. Papier à lettre entouré d'une frise de feuillage. Traits au crayon sur lesquels s'appuie une écriture d'enfant sans majuscules. Nous les rétablissons ici.

63 Voir la lettre de Jules à Juliette Marcille du $1^{\text {er }}$ janvier 1863, qui accompagne une caisse de jouets (Lettres (1885), op. cit., p. 222-223).

64. C'est le séjour évoqué dans le Journal du 17 décembre 1863.

65. Une lettre de Jules du 27 janvier 1864 remercie pour l'envoi de deux essuie-plumes (Lettres (1885), op. cit., p. 239-240). Dans La Maison d'un artiste, Edmond mentionne " un essuie-plumes brodé par les filles de madame Camille Marcille, quand elles étaient toutes perites filles " sur la table du cabinet de travail (op. cit., t. II, p. 67). 
image. Il ne faut pas que votre frère soit jaloux car je n'en ai qu'une.

Votre amie pour la vie

Juliette Marcille

[f. 65 : petite image de piété en dentelle de papier, avec ornements et nimbe de l'enfant Jésus dorés]

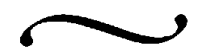

2. $[\mathrm{f} .66-68]^{66}$

Ah! Monsieur Jules, Ah! Monsieur Jules! Quelle tristesse! quelle tristesse! nous sommes tristes! Juliette est triste! Marguerite est triste! $\mathrm{Naco}^{67}$ est triste! Ma tante est triste! Clémentine, Mirza, Nounou sont tristes! Enfin c'est une tristesse! - des plus tristes!!

Ah! Monsieur Jules, Ah! Monsieur Jules! Plus de cache-cache, plus de colin-maillard, plus de promenades, plus de bonbons, plus de trente-et-un, plus de tartes, *plus de cigarettes, - pas même d'Artidors ${ }^{68}$ ! -* Ah! Monsieur Jules, Ah! Monsieur Jules!

Un silence partout! silence au dedans, silence au[-]dehors, silence dans la campagne, silence dans l'atelier, silence à table, silence dans les corridors, silence sur toute la ligne! *On n'entend plus que deux fois par jour ce refrain navrant - en classe! Mesdemoiselles en classe!!! - et puis c'est tout!* - Ah! Monsieur Jules, Ah! Monsieur Jules!!!!

Mon oncle fait le portrait de mon père, on va voir les Grandet! on lit le voyage Loin de Paris -, on va dîner à Gourdez! on reçoit la visite d'un curé! de deux curés! de trois curés! - *et l'[A]fricaine est toujours malade*.

Ah! Monsieur Jules, Ah! Monsieur Jules!!!

66. Cettc lettre est donnée en note des Lettres de Jules de Goncourt, publiées par Edmond en 1885 (op. cit., p. 240-242). Nous avons rétabli entre les signes * les passages supprimés dans certe publication.

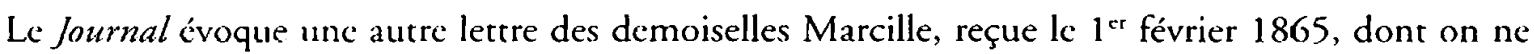
trouve pas trace dans les volumes de la Correspondance adressée aux Goncourt.

67. C'est probablement le surnom de la plus perite, Jane. Marie, la fille d'Eudoxe, rédige la lettre. Juliette ct Margucrite sont respectivement l'aînée et la cadette de Camille Marcille.

68. P'cu lisible. 
Le matin on passe encore doucement devant votre porte, croyant que vous dormez toujours ${ }^{(9)}$ ! - mais à dix heures vous dormez encore! et puis à midi encore! et puis le soir encore! - et puis la nuit encore - et puis toujours et puis toujours! -

Ah! Monsieur Jules! Ah! Monsieur Jules! ! ! -

Dans la journée on travaille - sans travailler! Car on croit toujours vous entendre vous écrier : - En classe, mesdemoiselles, en classe! Mais hélas! vaine attente! et pendant qu'on prête une oreille, quatre oreilles, six oreilles, huit oreilles, la plume tombe des mains, l'encrier tombe par terre, le cahier tombe sur l'encrier - et les larmes nous tombent des yeux! - !!

Ah! Monsieur Jules! Ah! Monsieur Jules!!!!

Le soir le fricandeau est sans charmes et sans champignons! On se verse à boire soi-même; on ne mange plus de plum-pudding, on ne s'assoit plus sous le feuillage, on fait une promenade * potagère* le long des plans d'oignons et des bordures d'oseille; puis on prend, en rentrant, une mortelle tapisserie - et on se couche à huit heures!!

Ah! Monsieur Jules! Ah! Monsieur Jules!!!!

La nuit est plus lugubre encore car il fait noir, car il fait noir... On est agité, on dégringole de son lit. On rêve dictée, analyse, catéchisme et chronologie; puis tout d'un coup on se réveille, ayant entendu $\mathrm{M}^{\text {lle }}$ Berthe appeler d'une voix terrible : En classe, mesdemoiselles, en classe!

Ah! Monsieur Jules! Ah! Monsieur Jules!

Écrivez-nous, monsieur Jules, *écrivez-nous!* Écrivez-nous à toutes ensemble, écrivez-nous à chacune en particulier. N'oubliez pas notre pantin ${ }^{70}$. N'oubliez pas le trente-et-un, *n'oubliez pas Artidor*. N'oubliez pas Oisème. N'oubliez rien! n'oubliez rien! Car *Monsieur Jules, ${ }^{*}$ tout pense à vous, tout vous aime, tout vous regrette, tout vous désire.

Ah! Monsieur Jules! ! !!

Ah! Monsieur Jules! ! ! ! ! ! !!

Tout vous embrasse,

69 Remplacé par " craignant que vous dormiez toujours " dans les Letrres de 1885.

70. Edmond ajoute entre parenthèses : "un pantin dessiné er gravé un jour de pluie et qui fit plus tard le frontispice des eaux-fortes de mon frère ". Voir J. de Goncourt, Eaux-fortes, notice et catalogue de Philippe Burty, Librairie de l'Arr, A. Ballue, C. Delagrave, 1876. 
Vos amies pour la vie, Marie Marcillexe "Marcille (C. et E.) ", Juliette Marcillexe "Marcille (C. et E.)", Marguerite Marcillexe «Marcille (C. et E.)».

Mon gros, je t'aime.

Nacotier. Oisème, ce mercredi ${ }^{*} 5^{*}$ juillet $1865^{71}$

71. Lettre postérieure au séjour des Goncourt à Oisème évoqué dans le Journalà la date du 27 juin 1865. 